\title{
STATISTICAL INFERENCE FOR KUMARASWAMY-EXPONENTIAL DISTRIBUTION BASED ON PROGRESSIVE TYPE-II CENSORED DATA WITH BINOMIAL REMOVALS
}

\author{
RAKHI MOHAN ${ }^{\star}$ \\ Department of Statistics, University of Kerala, Trivandrum, India \\ Email: rakhiammalu@gmail.com \\ MANOJ CHACKO \\ Department of Statistics, University of Kerala, Trivandrum, India \\ Email: manojchacko02@gmail.com
}

\begin{abstract}
SUMMARY
In this paper, estimation of parameters of Kumaraswamy-exponential distribution with shape parameters $\alpha$ and $\beta$ is considered based on a progressively type-II censored sample with binomial removals. Together with the unknown parameters, the removal probability $p$ is also estimated. Bayes estimators are obtained using different loss functions such as squared error, LINEX loss function and entropy loss function. All Bayesian estimates are compared with the corresponding maximum likelihood estimates numerically in terms of their bias and mean square error values and found that Bayes estimators perform better than MLE's for $\beta$ and $p$ and MLEs perform better than Bayes estimators for $\alpha$. A real data set is also used for illustration.
\end{abstract}

Keywords and phrases: Kumaraswamy-exponential distribution; Progressive type-II censoring; Binomial removals; Bayes estimates; MCMC method

\section{Introduction}

Censored sampling is common in life-testing experiments and it arises usually due to the failure of experimenter to observe failure times of all units on a life-test. In medical and industrial applications, researchers have to treat censored data because they usually do not have sufficient time to observe the lifetime of all subject in the study. There are different censoring schemes; for detail see, Lawless (2003). The most common censoring schemes are type-I and type-II censoring schemes. One of the drawbacks of the conventional type-I or type-II schemes is that they do not permit the flexibility of removal of units at points other than the terminal points of the experiment. This method is considered to be very efficient in terms of cost and time especially when the items/subjects being tested are very expensive (see Balakrishnan and Aggarwala, 2000).

The progressive type-II censoring scheme is defined as follows. Let $X_{1}, \ldots, X_{n}$ be independent and identically distributed (i.i.d.) random lifetimes of $n$ items. At the time of the first failure,

\footnotetext{
* Corresponding author

(C) Institute of Statistical Research and Training (ISRT), University of Dhaka, Dhaka 1000, Bangladesh.
} 
noted as $X_{1: m: n}, R_{1}$ units are randomly removed from $(n-1)$ surviving units. Similarly at the time of the second failure, noted as $X_{2: m: n}, R_{2}$ units from the $\left(n-R_{1}-2\right)$ units are randomly removed. This process continuous until, at the time of the $\mathrm{m}^{\text {th }}$ observed failure, the remaining ( $\left.n-m-R_{1}-R_{2}-\cdots-R_{m-1}\right)$ units are all removed from the experiment.

Usually, in progressive type-II censoring, the schemes $R_{1}, R_{2}, \ldots, R_{m}$ are all pre-fixed. However, random occurrence of these numbers may be seen in some practical situations. For example, in some reliability experiments, an experimenter might decide against testing of some of the units as it might be inappropriate or too dangerous to carry out the testing. In such cases, the removal of units after each failure tends to exhibit a random pattern. This leads to progressive censoring with random removals. In this paper, our assumption is that the units to be removed at the $i^{\text {th }}$ failure is random and the random removal $R_{i}$ follows a binomial distribution with parameter $p$. It means that unit leaves with equal probability $p$ and the probability of $R_{i}$ units leaving after the occurrence of $i^{\text {th }}$ failure for $i=1,2, \ldots, m$ is

$$
P\left(R_{1}=r_{1}\right)=\left(\begin{array}{c}
n-m \\
r_{1}
\end{array}\right) p^{r_{1}}(1-p)^{n-m-r_{1}}
$$

and for $i=2, \ldots, m$

$$
P\left(R_{i}=r_{i} \mid R_{i-1}=r_{i-1}, \ldots, R_{1}=r_{1}\right)=\left(\begin{array}{c}
n-m-\sum_{j=1}^{i-1} r_{j} \\
r_{i}
\end{array}\right) p^{r_{i}}(1-p)^{n-m-\sum_{j=1}^{i-1} r_{j}}
$$

where $0 \leq r_{i} \leq n-m-\sum_{j=1}^{i-1} r_{j}$. Furthermore, we assume that $R_{i}$ is independent of $X_{i}$ for all $i$. The schematic representation of the progressive type-II censoring with binomial removals is illustrated in Table 1.

Table 1: schematic representation of the progressive type-II censoring with binomial removals

\begin{tabular}{lllll}
\hline Process & The number in life testing & Failures & Binomial removals & Remains \\
\hline 1 & $n$ & 1 & $R_{1} \sim B(n-m, p)$ & $n-1-R_{1}$ \\
2 & $n-1-R_{1}$ & 1 & $R_{2} \sim B\left(n-m-R_{1}, p\right)$ & $n-2-R_{1}-R_{2}$ \\
$\ldots$ & $\ldots$ & $\ldots$ & $\ldots$ & $\ldots$ \\
$m-1$ & $n-(m-2)-\sum_{j=1}^{m-2} R_{j}$ & 1 & $R_{m-1} \sim B\left(n-m-\sum_{j=1}^{m-2} R_{j}, p\right)$ & $n-(m-1)-\sum_{j=1}^{m-1} R_{j}$ \\
$m$ & $n-(m-1)-\sum_{j=1}^{m-1} R_{j}$ & 1 & $R_{m}=n-m-\sum_{j=1}^{m-1} R_{j}$ & 0 \\
\hline
\end{tabular}

The joint distribution of $X=\left(X_{1: m: n}, X_{2: m: n}, \ldots, X_{m: m: n}\right)$ and $R=\left(R_{1}, R_{2}, \ldots, R_{m}\right)$ is obtained as

$$
f_{X, R}\left(x_{1: m: n}, x_{2: m: n}, \ldots, x_{m: m: n}, r \mid p\right)=f_{X}\left(x_{1: m: n}, x_{2: m: n}, \ldots, x_{m: m: n}\right) P(r \mid p),
$$


where

$$
f_{X}\left(x_{1: m: n}, x_{2: m: n}, \ldots, x_{m: m: n}\right)=C \prod_{i=1}^{m} f_{X_{i: m: n}}\left(x_{i: m: n}\right)\left\{1-F_{X_{i: m: n}}\left(x_{i: m: n}\right)\right\}^{r_{i}},
$$

$r=\left(r_{1}, r_{2}, \ldots, r_{m}\right), C$ is a constant defined as

$$
C=n\left(n-r_{1}-1\right)\left(n-r_{1}-r_{2}-2\right) \cdots\left(n-r_{1}-r_{2}\right) \cdots\left(n-r_{1}-r_{2}-\cdots-r_{m-1}-m+1\right)
$$

and $P(r \mid p)$ is the joint probability distribution of $R=\left(R_{1}, R_{2}, \ldots, R_{m}\right)$ defined as

$$
P(r \mid p)=P\left(R_{m}=r_{m} \mid R_{m-1}=r_{m-1}, \ldots, R_{1}=r_{1}\right) P\left(R_{2}=r_{2} \mid R_{1}=r_{1}\right) P\left(R_{1}=r_{1}\right) .
$$

Therefore from (1.1) and (1.2), we have

$$
P(r \mid p)=\frac{(n-m) !}{\prod_{j=1}^{m-1} r_{j} !\left(n-m-\sum_{j=1}^{m-1} r_{j}\right) !} p^{\sum_{j=1}^{m-1} r_{j}}(1-p)^{(m-1)(n-m)-\sum_{j=1}^{m-1}(m-j) r_{j} !} .
$$

Statistical inference on different lifetime distributions under progressive censoring with random removals were considered by several authors. Yuen and Tse (1996) considered parametric estimation for Weibull distribution under progressive censoring with random removals. Tse et al. (2000) considered statistical analysis for Weibull distributed lifetime data under type-II progressive censoring with binomial removals. Wu and Chang (2003) and Wu et al. (2004) discussed inference in the Pareto distribution based on progressive type-II censoring with random removals. Amin (2008) discussed Bayesian inference procedures of the Pareto distribution under progressive censoring with binomial removals. Al-Zahrani (2012) derived the maximum likelihood estimators of the Generalized Pareto distribution under progressive censoring with binomial removals. Azimi and Yaghmaei (2013) considered the Bayesian estimation based on Rayleigh distribution under progressive type-II censored data with binomial removals. Feroze and El-Batal (2013) derived the maximum likelihood estimators of the Kumaraswamy distribution under progressive type-II censored data with random removals. Azimi et al. (2014) discussed statistical inference procedures of the Pareto distribution using progressive type-II censoring data with binomial removals. Chacko and Mohan (2018) discussed statistical inference for Gompertz distribution under progressive type-II censoring with binomial removals.

In this paper, we consider progressive type-II censored sample taken from a Kumaraswamyexponential distribution with probability density function (pdf) given by

$$
f(x \mid \alpha, \beta)=\alpha \beta e^{-x}\left(1-e^{-x}\right)^{\alpha-1}\left(1-\left(1-e^{-x}\right)^{\alpha}\right)^{\beta-1}, x>0,
$$

and cumulative distribution function (cdf) given by

$$
F(x \mid \alpha, \beta)=1-\left(1-\left(1-e^{-x}\right)^{\alpha}\right)^{\beta}, x>0,
$$

where $\alpha>0, \beta>0$ are two shape parameters. 
Through out the paper we use the notation $\operatorname{KE}(\alpha, \beta)$ to denote Kumaraswamy-Exponential distribution with shape parameters $\alpha$ and $\beta$. KE distribution is a generalization of the Exponential distribution which is developed as a model for problems in environmental studies and survival analysis. Recently several authors have studied the Kumaraswamy distribution and its Generalizations (see Mohammed, 2014; de Araujo Rodrigues and Silva, 2015). Nadarajah et al. (2012) have studied general results for the Kumaraswamy-G distribution and discussed its properties. Elbatal (2013) introduced the Kumaraswamy linear exponential distribution and obtained its distributional properties. Lemonte et al. (2013) considered an exponentiated Kumaraswamy distribution and studied its properties.

This paper is organised as follows. In Section 2, maximum likelihood estimates of $\alpha, \beta$ and $p$ are obtained. The asymptotic variance-covariance matrix of the estimates is also obtained in this section. In Section 3, Bayes estimates for $\alpha, \beta$ and $p$ are obtained for different loss functions such as squared error, LINEX and general entropy. In Section 4, a simulation study is performed for analyzing the properties of different estimators developed in this paper. In Section 5, we illustrate the estimation procedure using a real data. Finally, a conclusion is given in Section 6.

\section{Maximum likelihood Estimation}

Let $X_{1: m: n}, X_{2: m: n}, \ldots, X_{m: m: n}, 1 \leq m \leq n$ be a progressively type-II censored sample observed from a life test involving $n$ units taken from a $\mathrm{KE}(\alpha, \beta)$ distribution and $\left(R_{1}, R_{2}, \ldots, R_{m}\right)$ being the censoring scheme with probability distribution defined in (1.5). Then the joint pdf of $X=$ $\left(X_{1: m: n}, X_{2: m: n}, \ldots, X_{m: m: n}\right)$ and $R=\left(R_{1}, R_{2}, \ldots, R_{m}\right)$ is given by

$$
f_{X, R}\left(x_{1: m: n}, x_{2: m: n}, \ldots, x_{m: m: n}, r \mid p\right)=f_{X}\left(x_{1: m: n}, x_{2: m: n}, \ldots, x_{m: m: n}\right) P(r \mid p),
$$

where

$$
\begin{aligned}
& f_{\left(X_{1: m: n}, X_{2: m: n}, \ldots, X_{m: m: n}\right)}\left(x_{1: m: n}, x_{2: m: n}, \ldots, x_{m: m: n}\right) \\
& \quad=C \alpha^{m} \beta^{m} \prod_{i=1}^{m} e^{-x_{i: m: n}}\left(1-e^{-x_{i: m: n}}\right)^{\alpha-1 .}\left[1-\left(1-e^{-x_{i: m: n}}\right)^{\alpha}\right]^{\beta\left(r_{i}+1\right)-1},
\end{aligned}
$$

$P(r \mid p)$ is as given in (1.5) and $C$ is as given in (1.4).

Then the likelihood function of $\alpha, \beta$ and $p$ is given by

$$
\begin{aligned}
L(\alpha, \beta, p \mid x, r) & \propto \alpha^{m} \beta^{m} \prod_{i=1}^{m} e^{-x_{i: m: n}}\left(1-e^{-x_{i: m: n}}\right)^{\alpha-1} \\
& \times\left[1-\left(1-e^{-x_{i: m: n}}\right)^{\alpha}\right]^{\beta\left(r_{i}+1\right)-1} p^{\sum_{j=1}^{m-1} r_{j}}(1-p)^{(m-1)(n-m)-\sum_{j=1}^{m-1}(m-j) r_{j}}
\end{aligned}
$$


Thus the log-likelihood function is given by

$$
\begin{aligned}
\log L(\alpha, \beta, p \mid x, r) & =m \log \alpha+m \log \beta-\sum_{i=1}^{m} x_{i: m: n}+(\alpha-1) \sum_{i=1}^{m} \log \left(1-e^{-x_{i: m: n}}\right) \\
& +\sum_{i=1}^{m}\left\{\beta\left(r_{i}+1\right)-1\right\} \log \left[1-\left(1-e^{-x_{i: m: n}}\right)^{\alpha}\right]+\left(\sum_{j=1}^{m-1} r_{j}\right) \log p \\
& +\left((m-1)(n-m)-\sum_{j=1}^{m-1}(m-j) r_{j}\right) \log (1-p)
\end{aligned}
$$

Thus we have

$$
\begin{aligned}
\frac{\partial \log L}{\partial \alpha}= & \frac{m}{\alpha}+\sum_{i=1}^{m} \log \left(1-e^{-x_{i: m: n}}\right) \\
& -\sum_{i=1}^{m} \frac{\left\{\beta\left(r_{i}+1\right)-1\right\}\left(1-e^{-x_{i: m: n}}\right)^{\alpha} \log \left(1-e^{-x_{i: m: n}}\right)}{\left[1-\left(1-e^{-x_{i: m: n}}\right)^{\alpha}\right]} \\
\frac{\partial \log L}{\partial \beta}= & \frac{m}{\beta}+\sum_{i=1}^{m}\left(r_{i}+1\right) \log \left[1-\left(1-e^{-x_{i: m: n}}\right)^{\alpha}\right. \\
\frac{\partial \log L}{\partial p}= & \frac{\sum_{j=1}^{m-1} r_{j}}{p}-\frac{(m-1)(n-m)-\sum_{j=1}^{m-1}(m-j) r_{j}}{1-p} .
\end{aligned}
$$

The maximum likelihood estimators of the parameters $\alpha, \beta$ and $p$ respectively can then be obtained as the solution of the following normal equations

$$
\begin{aligned}
& \frac{m}{\alpha}+\sum_{i=1}^{m} \log \left(1-e^{-x_{i: m: n}}\right) \\
&-\sum_{i=1}^{m} \frac{\left\{\beta\left(r_{i}+1\right)-1\right\}\left(1-e^{-x_{i: m: n}}\right)^{\alpha} \log \left(1-e^{-x_{i: m: n}}\right)}{\left[1-\left(1-e^{-x_{i: m: n}}\right)^{\alpha}\right]}=0 \\
& \frac{m}{\beta}+\sum_{i=1}^{m}\left(r_{i}+1\right) \log \left[1-\left(1-e^{-x_{i: m: n}}\right)^{\alpha}\right]=0 \\
& \frac{\sum_{j=1}^{m-1} r_{j}}{p}-\frac{(m-1)(n-m)-\sum_{j=1}^{m-1}(m-j) r_{j}}{1-p}=0 .
\end{aligned}
$$

Thus from (2.5) and (2.8), we have

$$
\beta=-\frac{m}{\sum_{i=1}^{m}\left(r_{i}+1\right) \log \left[1-\left(1-e^{-x_{i: m: n}}\right)^{\alpha}\right]} .
$$


On substituting (2.10) into (2.7), we get

$$
\begin{aligned}
& \frac{m}{\alpha}+\sum_{i=1}^{m} \log \left(1-e^{-x_{i: m: n}}\right) \\
& +\sum_{i=1}^{m}\left\{\frac{m\left(r_{i}+1\right)}{\sum_{i=1}^{m}\left(r_{i}+1\right) \log \left[1-\left(1-e^{-x_{i: m: n}}\right)^{\alpha}\right]}+1\right\} \frac{\left(1-e^{-x_{i: m: n}}\right)^{\alpha} \log \left(1-e^{-x_{i: m: n}}\right)}{\left[1-\left(1-e^{-x_{i: m: n}}\right)^{\alpha}\right]}=0 .
\end{aligned}
$$

The maximum likelihood estimator $\hat{\alpha}$ of $\alpha$ can be obtained as solution of the nonlinear equation of the form $g(\alpha)=\alpha$, where

$$
\begin{aligned}
g(\alpha)=- & m\left(\sum_{i=1}^{m} \log \left(1-e^{-x_{i: m: n}}\right)-\sum_{i=1}^{m}\left[\frac{m\left(1+r_{i}\right)}{\sum_{i=1}^{m}\left(1+r_{i}\right) \log \left[1-\left(1-e^{-x_{i: m: n}}\right)^{\alpha}\right]}-1\right]\right. \\
& \left.\times \frac{\left(1-e^{-x_{i: m: n}}\right)^{\alpha} \log \left(1-e^{-x_{i: m: n}}\right)}{\left[1-\left(1-e^{-x_{i: m: n}}\right)^{\alpha}\right]}\right)^{-1} .
\end{aligned}
$$

Let $\hat{\alpha}$ be the ML estimator of $\alpha$ by solving the non linear equation $g(\alpha)=\alpha$ and then by using (2.10), the ML estimator of $\beta$ will be given by

$$
\hat{\beta}=-\frac{m}{\sum_{i=1}^{m}\left(1+r_{i}\right) \log \left[1-\left(1-e^{-x_{i: m: n}}\right)^{\hat{\alpha}}\right]} .
$$

Also from (2.6) and (2.9), we have the ML estimator of $p$ as

$$
\hat{p}=\frac{\sum_{j=1}^{m-1} r_{j}}{(m-1)(n-m)-\sum_{j=1}^{m-1}(m-j) r_{j}+\sum_{j=1}^{m-1} r_{j}} .
$$

\subsection{Interval Estimation}

In this section, the appropriate confidence intervals of the parameters based on asymptotic distributions of ML of the parameters $\alpha, \beta$ and $p$ are obtained. Based on progressive censored samples, the elements of the Fisher information matrix for the parameters of the KE distribution have been derived explicitly. The Fisher information matrix can be defined as

$$
I=-E\left[\begin{array}{ccc}
\frac{\partial^{2} \log L}{\partial \alpha^{2}} & \frac{\partial^{2} \log L}{\partial \alpha \partial \beta} & \frac{\partial^{2} \log L}{\partial \alpha \partial p} \\
\frac{\partial^{2} \log L}{\partial \beta \partial \alpha} & \frac{\partial^{2} \log L}{\partial \beta^{2}} & \frac{\partial^{2} \ln L}{\partial \beta \partial p} \\
\frac{\partial^{2} \log L}{\partial p \partial \alpha} & \frac{\partial^{2} \log L}{\partial p \partial \beta} & \frac{\partial^{2} \ln L}{\partial p^{2}}
\end{array}\right] .
$$


As the exact mathematical expressions for the above expectations are difficult to obtain, we give the approximate (observed) information matrix, which is obtained by dropping the expectation operator $E$. It can be written as

$$
I=-\left[\begin{array}{ccc}
\frac{\partial^{2} \log L}{\partial \alpha^{2}} & \frac{\partial^{2} \log L}{\partial \alpha \partial \beta} & \frac{\partial^{2} \log L}{\partial \alpha \partial p} \\
\frac{\partial^{2} \log L}{\partial \beta \partial \alpha} & \frac{\partial^{2} \log L}{\partial \beta^{2}} & \frac{\partial^{2} \log L}{\partial \beta \partial p} \\
\frac{\partial^{2} \ln L}{\partial p \partial \alpha} & \frac{\partial^{2} \log L}{\partial p \partial \beta} & \frac{\partial^{2} \log L}{\partial p^{2}}
\end{array}\right]=\left[\begin{array}{ccc}
I_{\alpha \alpha} & I_{\alpha \beta} & I_{\alpha p} \\
I_{\beta \alpha} & I_{\beta \beta} & I_{\beta p} \\
I_{p \alpha} & I_{p \beta} & I_{p p}
\end{array}\right],
$$

where

$$
\begin{aligned}
& I_{\alpha \alpha}=-\frac{m}{\alpha^{2}}-\sum_{i=1}^{m}\left\{\beta\left(r_{i}+1\right)-1\right\}\left[\log \left(1-e^{-x_{i: m: n}}\right)\right]^{2}\left\{\frac{\left(1-e^{-x_{i: m: n}}\right)^{\alpha}}{\left[1-\left(1-e^{-x_{i: m: n}}\right)^{\alpha}\right]^{2}}\right\}, \\
& I_{\alpha \beta}=I_{\beta \alpha}=-\sum_{i=1}^{m} \frac{\left(r_{i}+1\right)\left(1-e^{-x_{i: m: n}}\right)^{\alpha} \log \left(1-e^{-x_{i: m: n}}\right)}{\left[1-\left(1-e^{-x_{i: m: n}}\right)^{\alpha}\right]}, \\
& I_{\beta \beta}=-\frac{m}{\beta^{2}}, I_{p p}=\frac{\sum_{i=1}^{m-1} r_{i}}{p^{2}}+\frac{(m-1)(n-m)-\sum_{i=1}^{m-1}(m-i) r_{i}}{(1-p)^{2}}, \\
& I_{\alpha p}=I_{p \alpha}=I_{\beta p}=I_{p \beta}=0 .
\end{aligned}
$$

Therefore the variance-covariance matrix may be approximated as

$$
V=\left(\begin{array}{ccc}
V_{\alpha \alpha} & V_{\alpha \beta} & 0 \\
V_{\beta \alpha} & V_{\beta \beta} & 0 \\
0 & 0 & V_{p p}
\end{array}\right)=\left(\begin{array}{ccc}
I_{\alpha \alpha} & I_{\alpha \beta} & 0 \\
I_{\beta \alpha} & I_{\beta \beta} & 0 \\
0 & 0 & I_{p p}
\end{array}\right)^{-1}
$$

Thus the asymptotic distribution of the MLEs can be written as follows (see, Miller (1981)),

$$
[(\hat{\alpha}-\alpha),(\hat{\beta}-\beta),(\hat{p}-p)] \longrightarrow N_{3}(0, V) .
$$

Since $V$ involves the parameters $\alpha, \beta$ and $p$, by replacing the parameters by the corresponding MLE's, estimate of $V$ can be obtained, which is denoted by $\hat{V}$. Approximate $100(1-\vartheta) \%$ confidence intervals for $\alpha, \beta$ and $p$ are determined by using (2.15), respectively as

$$
\hat{\alpha} \pm Z_{\vartheta / 2} \sqrt{\hat{V}_{\alpha \alpha}}, \hat{\beta} \pm Z_{\vartheta / 2} \sqrt{\hat{V}_{\beta \beta}} \text { and } \hat{p} \pm Z_{\vartheta / 2} \sqrt{\hat{V}_{p p}}
$$

where $Z_{\vartheta}$ is the upper $100 \vartheta^{\text {th }}$ percentile of the standard normal distribution.

\section{Bayesian estimation}

In this section, we consider the Bayes estimators of the parameters $\alpha, \beta$ and $p$ using progressively type-II censored data with binomial removals. The Bayes estimates are obtained using symmetric 
as well as asymmetric loss functions such as squared error, LINEX loss function and entropy loss function.

We assume that the prior distributions for $\alpha$ and $\beta$ follow $\operatorname{Gamma}\left(a_{1}, b_{1}\right)$ and $\operatorname{Gamma}\left(a_{2}, b_{2}\right)$ respectively, with pdfs given by

$$
\begin{aligned}
& \pi_{1}(\alpha) \propto \alpha^{a_{1}-1} e^{-b_{1} \alpha}, \alpha>0, a_{1}>0, b_{1}>0 \\
& \pi_{2}(\beta) \propto \beta^{a_{2}-1} e^{-b_{2} \beta}, \beta>0, a_{2}>0, b_{2}>0 .
\end{aligned}
$$

Here $a_{1}, b_{1}, a_{2}$ and $b_{2}$ are chosen to reflect prior knowledge about $\alpha$ and $\beta$. Independently from parameters $\alpha$ and $\beta, p$ has a beta prior distribution with parameters $a$ and $b$ of the form,

$$
\pi_{3}(p) \propto p^{a-1}(1-p)^{b-1}, 0<p<1, a>0, b>0 .
$$

Based on the priors $\pi_{1}(\alpha), \pi_{2}(\beta)$ and $\pi_{3}(p)$, the joint prior pdf of $(\alpha, \beta, p)$ is

$$
\begin{aligned}
\pi(\alpha, \beta, p) & \propto \pi_{1}(\alpha) \pi_{2}(\beta) \pi_{3}(p) \\
& \propto \alpha^{a_{1}-1} e^{-b_{1} \alpha} \beta^{a_{2}-1} e^{-b_{2} \beta} p^{a-1}(1-p)^{b-1} ; \alpha, \beta>0,0<p<1 .
\end{aligned}
$$

From (1.3) the likelihood function of $\alpha, \beta$ and $p$ is given by

$$
\begin{aligned}
L(\alpha, \beta, p \mid x, r) & \propto \alpha^{m} \beta^{m} \prod_{i=1}^{m} e^{-x_{i: m: n}}\left(1-e^{-x_{i: m: n}}\right)^{\alpha-1}\left[1-\left(1-e^{-x_{i: m: n}}\right)^{\alpha}\right]^{\beta\left(r_{i}+1\right)-1} \\
& \times p^{\sum_{j=1}^{m-1} r_{j}}(1-p)^{(m-1)(n-m)-\sum_{j=1}^{m-1}(m-j) r_{j}}
\end{aligned}
$$

where $x=\left(x_{1: m: n}, x_{2: m: n}, \ldots, x_{m: m: n}\right)$ and $r=\left(r_{1}, r_{2}, \ldots, r_{m}\right)$.

Then the joint posterior distribution of $\alpha, \beta$ and $p$ is given by

$$
\pi^{*}(\alpha, \beta, p \mid x, r)=\frac{L(\alpha, \beta, p \mid x, r) \pi(\alpha, \beta, p)}{\int_{0}^{\infty} \int_{0}^{\infty} \int_{0}^{1} L(\alpha, \beta, p \mid x, r) \pi(\alpha, \beta, p) d p d \beta d \alpha} .
$$

The above equation can be written as

$$
\begin{aligned}
\pi^{*}(\alpha, \beta, p \mid x) & \propto \alpha^{m+a_{1}-1} \beta^{m+a_{2}-1} p^{a+\sum_{j=1}^{m-1} r_{j}-1}(1-p)^{b+(m-1)(n-m)-\sum_{j=1}^{m-1}(m-j) r_{j}-1} \\
& \times e^{-\alpha\left[b_{1}-\sum_{i=1}^{m} \log \left(1-e^{-x_{i: m}: n}\right)\right]} \prod_{i=1}^{m}\left[1-\left(1-e^{-x_{i: m: n}}\right)^{\alpha}\right]^{-1} \\
& \times e^{-\beta\left\{b_{2}-\sum_{i=1}^{m}\left(r_{i}+1\right) \log \left[1-\left(1-e^{-x_{i: m: n}}\right)^{\alpha}\right]\right\}}
\end{aligned}
$$

From (3.7), it is clear that it is not possible to get explicit forms for expectation of the marginal posterior distributions for each parameter. Hence we use MCMC method for sampling from posterior distributions and computing posterior quantities of interest. A Gibb's algorithm is required 
to decompose the joint posterior distribution into full conditional distributions for each parameter in the model, then sample from each one of these conditional distributions and compute the Bayes estimate of $\alpha, \beta$ and $p$.

The conditional density of $\alpha$, given $\beta, p$ and data is given by

$$
\begin{aligned}
\pi_{1}^{*}(\alpha \mid \beta, p, x) & \propto \alpha^{m+a_{1}-1} e^{-\alpha\left[b_{1}-\sum_{i=1}^{m} \log \left(1-e^{-x_{i: m: n}}\right)\right]} \prod_{i=1}^{m}\left[1-\left(1-e^{-x_{i: m: n}}\right)^{\alpha}\right]^{-1} \\
& \times e^{\beta \sum_{i=1}^{m}\left(r_{i}+1\right) \log \left[1-\left(1-e^{-x_{i: m: n}}\right)^{\alpha}\right]}
\end{aligned}
$$

Similarly, the conditional density of $\beta$, given $\alpha, p$ and data is given by

$$
\pi_{2}^{*}(\beta \mid \alpha, p, x) \propto \beta^{m+a_{2}-1} e^{-\beta\left\{b_{2}-\sum_{i=1}^{m}\left(r_{i}+1\right) \log \left[1-\left(1-e^{-x_{i: m: n}}\right)^{\alpha}\right]\right\}} .
$$

Again, the conditional density of $p$, given $\alpha, \beta$ and data is given by

$$
\pi_{3}^{*}(p \mid \alpha, \beta, x) \propto p^{a+\sum_{j=1}^{m-1} r_{j}-1}(1-p)^{b+(m-1)(n-m)-\sum_{j=1}^{m-1}(m-j) r_{j}-1} .
$$

From (3.9) we can see that the conditional distribution of $\beta$ given $\alpha, p$ and data follows

$$
\operatorname{Gamma}\left(m+a_{2}, b_{2}-\sum_{i=1}^{m}\left(r_{i}+1\right) \log \left[1-\left(1-e^{-x_{i: m: n}}\right)^{\alpha}\right]\right)
$$

Similarly, from (3.10) we can see that the conditional distribution of $p$ given $\alpha, \beta$ and data follows

$$
\operatorname{Beta}\left(a+\sum_{j=1}^{m-1} r_{j}, b+(m-1)(n-m)-\sum_{j=1}^{m-1}(m-j) r_{j}\right)
$$

Therefore one can easily generate samples from the posterior distributions of $\beta$ and $p$. But it is not possible to generate random variables from the posterior distribution of $\alpha$ given in (3.8) using standard random number generation methods, therefore we use Metropolis-Hastings (M-H) algorithm within the Gibbs sampling procedure to generate sample from (3.8). Since plot of (3.8) is similar to a normal plot we take normal proposal density for $\alpha$ for the M-H algorithm.

By setting initial values $\alpha^{(0)}, \beta^{(0)}$ and $p^{(0)}$, let $\alpha^{(j)}, \beta^{(j)}$ and $p^{(j)}, j=1, \ldots, N$ be the observations generated from (3.8), (3.9) and (3.10) respectively. Then, by taking the first $M$ iterations as burn-in period, the Bayes estimates of $\alpha, \beta$ and $p$ against different loss functions are as given below.

The Bayes estimates $\hat{\psi}_{S B}, \hat{\psi}_{L B}$ and $\hat{\psi}_{E B}$ of $\psi$ under squared error, LINEX loss function and 
entropy loss function, respectively, are obtained as follows:

$$
\begin{aligned}
& \hat{\psi}_{S B}=\frac{1}{N-M} \sum_{j=M+1}^{N} \psi^{(j)} \\
& \hat{\psi}_{L B}=-\frac{1}{h} \log \left(\frac{1}{N-M} \sum_{j=M+1}^{N} e^{-h \psi^{(j)}}\right) \\
& \hat{\psi}_{E B}=\left\{\frac{1}{N-M} \sum_{j=M+1}^{N}\left(\psi^{(j)}\right)^{-q}\right\}^{-\frac{1}{q}},
\end{aligned}
$$

where $\psi$ stands for $\alpha, \beta$ or $p$.

\section{Simulation Study}

In this section, a simulation is performed to study the behavior of different estimators for the parameters $\alpha, \beta$ and $p$. The performance of all estimators are compared numerically in terms of their bias and MSE values for different combinations of $n, m, \alpha, \beta$ and $p$. First we obtain the MLE's of $\alpha, \beta$ and $p$ using 500 generated samples. The bias and MSE for the MLE's of $\alpha, \beta$ and $p$ for $p=0.25,0.5$ and 0.75 are given in Tables 2, 3 and 4 respectively. For the simulation studies for Bayes estimators we took hyperparameters for the prior distributions of $\alpha$ and $\beta$ as $a_{1}=3, a_{2}=3, b_{1}=3, b_{2}=3$, $a=3$ and $b=3$. We have obtained the Bayes estimates for $\alpha, \beta$ and $p$ using MCMC method. For the MCMC method we do the following

1. For a given $n$ generate $m$ progressive type-II censored sample from $\operatorname{KE}(\alpha, \beta)$.

2. Calculate estimators of the parameters $\alpha, \beta$ and $p$ using MCMC method as describe below.

(a) Start with initial guess $\left(\alpha^{(0)}, \beta^{(0)}, p^{(0)}\right)$.

(b) Set $j=1$.

(c) Generate $\beta^{(j)}$ from

$$
\operatorname{Gamma}\left(m+a_{2}, b_{2}-\sum_{i=1}^{m}\left(r_{i}+1\right) \log \left[1-\left(1-e^{-x_{i: m: n}}\right)^{\alpha^{(j-1)}}\right]\right) .
$$

(d) Generate $p^{(j)}$ from

$$
\operatorname{Beta}\left(a+\sum_{j=1}^{m-1} r_{j}, b+(m-1)(n-m)-\sum_{j=1}^{m-1}(m-j) r_{j}\right) .
$$

(e) Using Metropolis-Hastings algorithm, generate $\alpha^{(j)}$ from (3.8) with the normal proposal density. 
(f) Set $j=j+1$.

(g) Repeat the steps from (c) to (f) for $N=50,000$ times.

(h) Calculate the Bayes estimators of the parameters $\alpha, \beta$ and $p$ under different functions by taking first 5000 iteration as burn-in period.

3. Repeat the steps 1 and 2 for 500 times.

4. Calculate the bias and MSE of all estimators.

The bias and MSE for the Bayes estimates of $\alpha$ under different loss functions for $p=0.25,0.5$ and 0.75 are given in Table 2. The bias and MSE for the estimates of $\beta$ under different loss functions for $p=0.25,0.5$ and 0.75 are given in Table 3. The bias and MSE for the estimates of $p$ under different loss functions for different choices of $p=0.25,0.5$ and 0.75 are given in Table 4. For evaluating Bayes estimators under LINEX loss function $L_{2}$ we take $h=1$ and entropy loss function $L_{3}$ we take $q=1$.

From the tables, we can see that for $\beta$ and $p$ the Bayes estimators have smaller bias and MSE than MLE's. But for $\alpha$ bias and MSE of MLE's are smaller than bias and MSE of Bayes estimators. From Table 3, one can see that the bias and MSE of Bayes estimators of $\beta$ under squared error loss function $\left(\hat{\beta}_{S B}\right)$ are smaller than bias and MSE of other estimators of $\beta$. From Table 4 , one can see that for $p \leq 0.5$ the bias and MSE of Bayes estimators of $p$ under entropy loss function $\left(\hat{\alpha}_{E B}\right)$ are smaller than bias and MSE of other estimators of $p$ and for $p>0.5$ Bayes estimators under squared error loss function $\left(\hat{p}_{S B}\right)$ have smaller bias and MSE than other estimators of $p$.

\section{Illustration using Real-Life Data}

In this section, we illustrate the inferential procedure using a real life data. For that we use a data set on active repair times (in hours) for an airborne transceiver, reported in Jorgensen (1982).

$0.50,0.60,0.60,0.70,0.70,0.70,0.80,0.80,1.00,1.00,1.00,1.00,1.10,1.30,1.50$ $1.50,1.50,1.50,2.00,2.00,2.20,2.50,2.70,3.00,3.00,3.30,4.00,4.00,4.50,4.70$, $5.00,5.40,5.40,7.00,7.50,8.80,9.00,10.20,22.00,24.50$.

To check for the goodness of fit we use the Anderson-Darling test (see, Stephens (1974)). We have computed the Anderson-Darling statistic for the data using 'DistributionFitTest' function in Mathematica and is obtained as 1.53775 . The corresponding $P$-Value is 0.167823 . Since the $P$ Value is quite high, we cannot reject the null hypothesis that the data are coming from the KE distribution.

From the data set we generated progressive type-II censored observation with binomial removals for different values of $p(p=0.25,0.5,0.75)$ and $m(m=10,15,20)$ and are given in Table 5. We have obtained the MLE's of $\alpha, \beta$ and $p$ and Bayes estimates of $\alpha, \beta$ and $p$ under squared error, LINEX and entropy loss functions are given in Table 6 . 
Table 2: Bias and MSE of the MLE $\hat{\alpha}$, Bayes estimates under squared error loss function $\hat{\alpha}_{S B}$, Bayes estimates under LINEX error loss function $\hat{\alpha}_{L B}$ and Bayes estimates under entropy loss function $\hat{\alpha}_{E B}$ for different choices $n, m, \alpha, \beta$ and $p=0.25,0.5$ and 0.75 .

\begin{tabular}{|c|c|c|c|c|c|c|c|c|c|c|c|c|}
\hline \multirow[t]{2}{*}{$p$} & \multirow[t]{2}{*}{$n$} & \multirow[t]{2}{*}{$m$} & \multirow[t]{2}{*}{$\alpha$} & \multirow[t]{2}{*}{$\beta$} & \multicolumn{2}{|c|}{$\hat{\alpha}$} & \multicolumn{2}{|c|}{$\hat{\alpha}_{S B}$} & \multicolumn{2}{|c|}{$\hat{\alpha}_{L B}$} & \multicolumn{2}{|c|}{$\hat{\alpha}_{E B}$} \\
\hline & & & & & Bias & MSE & Bias & MSE & Bias & MSE & Bias & MSE \\
\hline \multirow[t]{12}{*}{0.25} & \multirow[t]{6}{*}{30} & \multirow[t]{3}{*}{5} & 0.5 & 1 & 0.017 & 0.320 & 0.666 & 0.707 & 0.414 & 0.605 & 0.284 & 0.514 \\
\hline & & & 1 & 1.5 & 0.518 & 0.432 & -0.507 & 0.531 & -0.372 & 0.460 & -0.253 & 0.341 \\
\hline & & & 1 & 2 & 0.524 & 0.548 & -0.348 & 0.647 & -0.230 & 0.430 & -0.069 & 0.362 \\
\hline & & \multirow[t]{3}{*}{10} & 0.5 & 1 & -0.031 & 0.002 & 0.305 & 0.419 & 0.209 & 0.241 & 0.090 & 0.206 \\
\hline & & & 1 & 1.5 & 0.532 & 0.452 & -0.544 & 0.387 & -0.422 & 0.270 & -0.371 & 0.235 \\
\hline & & & 1 & 2 & 0.087 & 0.015 & -0.466 & 0.290 & -0.361 & 0.366 & -0.287 & 0.279 \\
\hline & \multirow[t]{6}{*}{50} & \multirow[t]{3}{*}{10} & 0.5 & 1 & 0.044 & 0.004 & 0.351 & 0.515 & 0.239 & 0.294 & 0.145 & 0.258 \\
\hline & & & 1 & 1.5 & 0.322 & 0.207 & -0.551 & 0.402 & -0.444 & 0.295 & -0.397 & 0.277 \\
\hline & & & 1 & 2 & 0.020 & 0.001 & -0.480 & 0.432 & -0.368 & 0.423 & -0.272 & 0.328 \\
\hline & & \multirow[t]{3}{*}{25} & 0.5 & 1 & 0.182 & 0.066 & 0.090 & 0.075 & -0.069 & 0.073 & 0.050 & 0.071 \\
\hline & & & 1 & 1.5 & -0.275 & 0.152 & -0.668 & 0.486 & -0.554 & 0.347 & -0.511 & 0.299 \\
\hline & & & 1 & 2 & -0.194 & 0.075 & -0.572 & 0.401 & -0.459 & 0.286 & -0.416 & 0.254 \\
\hline \multirow[t]{12}{*}{0.5} & \multirow[t]{6}{*}{30} & \multirow[t]{3}{*}{5} & 0.5 & 1 & 0.097 & 0.019 & 0.770 & 0.840 & 0.513 & 0.670 & 0.377 & 0.549 \\
\hline & & & 1 & 1.5 & 0.168 & 0.057 & -0.454 & 0.326 & -0.338 & 0.387 & -0.253 & 0.295 \\
\hline & & & 1 & 2 & 0.198 & 0.078 & -0.364 & 0.409 & -0.237 & 0.384 & -0.113 & 0.307 \\
\hline & & \multirow[t]{3}{*}{10} & 0.5 & 1 & 0.178 & 0.063 & 0.198 & 0.320 & 0.108 & 0.171 & 0.000 & 0.159 \\
\hline & & & 1 & 1.5 & -0.236 & 0.112 & -0.540 & 0.365 & -0.431 & 0.415 & -0.362 & 0.309 \\
\hline & & & 1 & 2 & 0.663 & 0.879 & -0.468 & 0.363 & -0.359 & 0.272 & -0.299 & 0.267 \\
\hline & \multirow[t]{6}{*}{50} & \multirow[t]{3}{*}{10} & 0.5 & 1 & 0.039 & 0.003 & 0.303 & 0.434 & 0.195 & 0.261 & 0.046 & 0.225 \\
\hline & & & 1 & 1.5 & 0.369 & 0.273 & -0.529 & 0.391 & -0.418 & 0.285 & -0.363 & 0.280 \\
\hline & & & 1 & 2 & 0.040 & 0.003 & -0.509 & 0.368 & -0.385 & 0.257 & -0.323 & 0.237 \\
\hline & & \multirow[t]{3}{*}{25} & 0.5 & 1 & -0.093 & 0.017 & 0.042 & 0.069 & -0.114 & 0.056 & -0.001 & 0.055 \\
\hline & & & 1 & 1.5 & 0.059 & 0.007 & -0.631 & 0.451 & -0.510 & 0.313 & -0.469 & 0.270 \\
\hline & & & 1 & 2 & -0.284 & 0.162 & -0.621 & 0.444 & -0.487 & 0.295 & -0.436 & 0.257 \\
\hline \multirow[t]{12}{*}{0.75} & 30 & 5 & 0.5 & 1 & 0.096 & 0.018 & 0.458 & 0.790 & 0.298 & 0.358 & 0.160 & 0.295 \\
\hline & & & 1 & 1.5 & 0.010 & 0.096 & -0.450 & 0.363 & -0.326 & 0.393 & -0.237 & 0.297 \\
\hline & & & 1 & 2 & 0.331 & 0.219 & -0.327 & 0.575 & -0.204 & 0.423 & -0.070 & 0.358 \\
\hline & & 10 & 0.5 & 1 & 0.025 & 0.001 & 0.127 & 0.179 & -0.066 & 0.115 & 0.063 & 0.115 \\
\hline & & & 1 & 1.5 & -0.125 & 0.031 & -0.584 & 0.428 & -0.476 & 0.314 & -0.429 & 0.287 \\
\hline & & & 1 & 2 & -0.147 & 0.043 & -0.467 & 0.397 & -0.365 & 0.382 & -0.286 & 0.312 \\
\hline & 50 & 10 & 0.5 & 1 & -0.132 & 0.035 & 0.160 & 0.166 & 0.104 & 0.124 & -0.011 & 0.113 \\
\hline & & & 1 & 1.5 & -0.290 & 0.168 & -0.548 & 0.388 & -0.427 & 0.270 & -0.378 & 0.238 \\
\hline & & & 1 & 2 & -0.073 & 0.011 & -0.471 & 0.370 & -0.362 & 0.324 & -0.289 & 0.280 \\
\hline & & 25 & 0.5 & 1 & 0.329 & 0.217 & 0.049 & 0.051 & -0.126 & 0.066 & -0.006 & 0.051 \\
\hline & & & 1 & 1.5 & -0.223 & 0.099 & -0.597 & 0.419 & -0.496 & 0.308 & -0.460 & 0.270 \\
\hline & & & 1 & 2 & 0.297 & 0.176 & -0.569 & 0.405 & -0.441 & 0.276 & -0.390 & 0.256 \\
\hline
\end{tabular}

\section{Conclusion}

In this paper, we considered a maximum likelihood estimation and Bayesian estimation of $\operatorname{KE}(\alpha, \beta)$ based on data under type-II progressive censoring scheme with binomial removals. The Bayes esti- 
Table 3: Bias and MSE of the MLE $\hat{\beta}$, Bayes estimates under squared error loss function $\hat{\beta}_{S B}$, Bayes estimates under LINEX error loss function $\hat{\beta}_{L B}$ and Bayes estimates under entropy loss function $\hat{\beta}_{E B}$ for different choices $n, m, \alpha, \beta$ and $p=0.25,0.5$ and 0.75 .

\begin{tabular}{|c|c|c|c|c|c|c|c|c|c|c|c|c|}
\hline \multirow[t]{2}{*}{$p$} & \multirow[t]{2}{*}{$n$} & \multirow[t]{2}{*}{$m$} & \multirow[t]{2}{*}{$\alpha$} & \multirow[t]{2}{*}{$\beta$} & \multicolumn{2}{|c|}{$\hat{\beta}$} & \multicolumn{2}{|c|}{$\hat{\beta}_{S B}$} & \multicolumn{2}{|c|}{$\hat{\beta}_{L B}$} & \multicolumn{2}{|c|}{$\hat{\beta}_{E B}$} \\
\hline & & & & & Bias & MSE & Bias & MSE & Bias & MSE & Bias & MSE \\
\hline \multirow[t]{12}{*}{0.25} & \multirow[t]{6}{*}{30} & \multirow[t]{3}{*}{5} & 0.5 & 1 & -0.514 & 0.491 & 0.046 & 0.363 & 0.181 & 0.393 & 0.424 & 0.687 \\
\hline & & & 1 & 1.5 & 0.953 & 0.977 & -0.584 & 0.596 & -0.710 & 0.687 & -0.863 & 0.927 \\
\hline & & & 1 & 2 & 0.965 & 0.975 & -0.888 & 0.976 & -0.948 & 0.977 & -0.983 & 0.979 \\
\hline & & \multirow[t]{3}{*}{10} & 0.5 & 1 & 0.670 & 0.574 & 0.106 & 0.382 & 0.211 & 0.415 & 0.441 & 0.802 \\
\hline & & & 1 & 1.5 & 0.971 & 0.994 & -0.552 & 0.493 & -0.659 & 0.577 & -0.780 & 0.752 \\
\hline & & & 1 & 2 & 0.958 & 0.934 & -0.805 & 0.929 & -0.989 & 0.929 & -0.903 & 0.967 \\
\hline & \multirow[t]{6}{*}{50} & \multirow[t]{3}{*}{10} & 0.5 & 1 & 0.474 & 0.449 & 0.195 & 0.531 & 0.276 & 0.569 & 0.578 & 0.919 \\
\hline & & & 1 & 1.5 & 0.977 & 0.929 & -0.625 & 0.610 & -0.733 & 0.692 & -0.869 & 0.909 \\
\hline & & & 1 & 2 & -0.553 & 0.611 & -0.890 & 0.959 & -0.986 & 0.987 & -0.931 & 0.995 \\
\hline & & \multirow[t]{3}{*}{25} & 0.5 & 1 & 0.960 & 0.944 & 0.151 & 0.300 & 0.030 & 0.188 & -0.055 & 0.190 \\
\hline & & & 1 & 1.5 & -0.417 & 0.348 & -0.658 & 0.500 & -0.747 & 0.619 & -0.857 & 0.796 \\
\hline & & & 1 & 2 & 0.701 & 0.983 & -0.855 & 0.975 & -0.994 & 0.995 & -0.996 & 0.989 \\
\hline \multirow[t]{12}{*}{0.5} & \multirow[t]{6}{*}{30} & \multirow[t]{3}{*}{5} & 0.5 & 1 & 0.944 & 0.906 & 0.104 & 0.341 & 0.250 & 0.619 & 0.480 & 0.290 \\
\hline & & & 1 & 1.5 & 0.987 & 0.913 & -0.541 & 0.572 & -0.667 & 0.644 & -0.806 & 0.849 \\
\hline & & & 1 & 2 & 0.965 & 0.933 & -0.845 & 0.900 & -0.917 & 0.988 & -0.950 & 0.989 \\
\hline & & \multirow[t]{3}{*}{10} & 0.5 & 1 & 0.676 & 0.562 & 0.026 & 0.230 & -0.082 & 0.236 & 0.213 & 0.467 \\
\hline & & & 1 & 1.5 & -0.559 & 0.518 & -0.522 & 0.561 & -0.644 & 0.607 & -0.756 & 0.763 \\
\hline & & & 1 & 2 & 0.685 & 0.938 & -0.754 & 0.930 & -0.930 & 0.953 & -0.937 & 0.939 \\
\hline & \multirow[t]{6}{*}{50} & \multirow[t]{3}{*}{10} & 0.5 & 1 & -0.252 & 0.127 & 0.020 & 0.402 & 0.161 & 0.428 & 0.444 & 0.897 \\
\hline & & & 1 & 1.5 & 0.975 & 0.975 & -0.572 & 0.607 & -0.691 & 0.668 & -0.810 & 0.847 \\
\hline & & & 1 & 2 & 0.622 & 0.599 & -0.897 & 0.912 & -0.920 & 0.945 & -0.969 & 0.966 \\
\hline & & \multirow[t]{3}{*}{25} & 0.5 & 1 & 0.643 & 0.524 & -0.042 & 0.126 & 0.063 & 0.142 & -0.136 & 0.143 \\
\hline & & & 1 & 1.5 & 0.562 & 0.453 & -0.572 & 0.412 & -0.657 & 0.518 & -0.769 & 0.678 \\
\hline & & & 1 & 2 & -0.641 & 0.821 & -0.866 & 0.917 & -0.936 & 0.973 & -0.962 & 0.993 \\
\hline \multirow[t]{12}{*}{0.75} & 30 & 5 & 0.5 & 1 & 0.810 & 0.931 & 0.014 & 0.264 & 0.136 & 0.283 & 0.332 & 0.481 \\
\hline & & & 1 & 1.5 & 0.939 & 0.965 & -0.540 & 0.547 & -0.654 & 0.623 & -0.786 & 0.812 \\
\hline & & & 1 & 2 & 0.997 & 0.957 & -0.870 & 0.921 & -0.935 & 0.934 & -0.976 & 0.947 \\
\hline & & 10 & 0.5 & 1 & 0.584 & 0.682 & -0.014 & 0.202 & -0.129 & 0.219 & 0.135 & 0.372 \\
\hline & & & 1 & 1.5 & -0.557 & 0.450 & -0.560 & 0.554 & -0.671 & 0.620 & -0.783 & 0.782 \\
\hline & & & 1 & 2 & -0.823 & 0.956 & -0.802 & 0.924 & -0.965 & 0.983 & -0.970 & 0.996 \\
\hline & 50 & 10 & 0.5 & 1 & 0.570 & 0.621 & 0.055 & 0.282 & -0.058 & 0.282 & 0.228 & 0.513 \\
\hline & & & 1 & 1.5 & -0.597 & 0.477 & -0.556 & 0.503 & -0.676 & 0.603 & -0.820 & 0.819 \\
\hline & & & 1 & 2 & -0.660 & 0.551 & -0.862 & 0.926 & -0.918 & 0.993 & -0.949 & 0.977 \\
\hline & & 25 & 0.5 & 1 & 0.956 & 0.943 & -0.033 & 0.116 & 0.071 & 0.163 & -0.129 & 0.132 \\
\hline & & & 1 & 1.5 & 0.653 & 0.060 & -0.588 & 0.458 & -0.659 & 0.544 & -0.751 & 0.674 \\
\hline & & & 1 & 2 & 0.524 & 0.500 & -0.743 & 0.942 & -0.911 & 0.990 & -0.917 & 0.945 \\
\hline
\end{tabular}

mates obtained using different loss functions such as squared error, LINEX and general entropy. To evaluate the Bayes estimates MCMC method has been applied. A simulation study was performed to evaluate the performance of the obtained estimators. The Bayes estimators for $\beta$ and $p$ perform 
Table 4: Bias and MSE of the MLE $\hat{p}$, Bayes estimates under squared error loss function $\hat{p}_{S B}$, Bayes estimates under LINEX error loss function $\hat{p}_{L B}$ and Bayes estimates under entropy loss function $\hat{p}_{E B}$ for different choices $n, m, \alpha, \beta$ and $p=0.25,0.5$ and 0.75 .

\begin{tabular}{|c|c|c|c|c|c|c|c|c|c|c|c|c|}
\hline \multirow[t]{2}{*}{$p$} & \multirow[t]{2}{*}{$n$} & \multirow[t]{2}{*}{$m$} & \multirow[t]{2}{*}{$\alpha$} & \multirow[t]{2}{*}{$\beta$} & \multicolumn{2}{|c|}{$\hat{p}$} & \multicolumn{2}{|c|}{$\hat{p}_{S B}$} & \multicolumn{2}{|c|}{$\hat{p}_{L B}$} & \multicolumn{2}{|c|}{$\hat{p}_{E B}$} \\
\hline & & & & & Bias & MSE & Bias & MSE & Bias & MSE & Bias & MSE \\
\hline \multirow[t]{12}{*}{0.25} & \multirow[t]{6}{*}{30} & \multirow[t]{3}{*}{5} & 0.5 & 1 & 0.083 & 0.014 & 0.035 & 0.001 & 0.032 & 0.001 & 0.016 & 0.000 \\
\hline & & & 1 & 1.5 & 0.047 & 0.004 & 0.042 & 0.002 & 0.038 & 0.002 & 0.024 & 0.001 \\
\hline & & & 1 & 2 & 0.011 & 0.000 & 0.045 & 0.002 & 0.042 & 0.002 & 0.027 & 0.001 \\
\hline & & \multirow[t]{3}{*}{10} & 0.5 & 1 & 0.015 & 0.000 & 0.099 & 0.010 & 0.097 & 0.010 & 0.086 & 0.008 \\
\hline & & & 1 & 1.5 & -0.019 & 0.001 & 0.042 & 0.002 & 0.039 & 0.002 & 0.025 & 0.001 \\
\hline & & & 1 & 2 & -0.052 & 0.005 & 0.021 & 0.001 & 0.017 & 0.000 & 0.001 & 0.000 \\
\hline & \multirow[t]{6}{*}{50} & \multirow[t]{3}{*}{10} & 0.5 & 1 & -0.018 & 0.001 & 0.015 & 0.000 & 0.012 & 0.000 & 0.000 & 0.000 \\
\hline & & & 1 & 1.5 & -0.044 & 0.004 & 0.031 & 0.001 & 0.028 & 0.001 & 0.017 & 0.000 \\
\hline & & & 1 & 2 & 0.029 & 0.002 & 0.031 & 0.001 & 0.028 & 0.001 & 0.017 & 0.000 \\
\hline & & \multirow[t]{3}{*}{25} & 0.5 & 1 & 0.071 & 0.010 & 0.017 & 0.000 & 0.014 & 0.000 & 0.000 & 0.000 \\
\hline & & & 1 & 1.5 & 0.031 & 0.002 & 0.011 & 0.000 & 0.009 & 0.000 & -0.007 & 0.000 \\
\hline & & & 1 & 2 & 0.010 & 0.000 & 0.007 & 0.000 & 0.010 & 0.000 & -0.004 & 0.000 \\
\hline \multirow[t]{12}{*}{0.5} & \multirow[t]{6}{*}{30} & \multirow[t]{3}{*}{5} & 0.5 & 1 & 0.100 & 0.020 & -0.088 & 0.008 & -0.081 & 0.007 & -0.079 & 0.006 \\
\hline & & & 1 & 1.5 & 0.058 & 0.007 & 0.100 & 0.010 & 0.097 & 0.010 & 0.091 & 0.009 \\
\hline & & & 1 & 2 & -0.132 & 0.035 & -0.007 & 0.000 & -0.014 & 0.001 & -0.005 & 0.000 \\
\hline & & \multirow[t]{3}{*}{10} & 0.5 & 1 & 0.056 & 0.006 & -0.062 & 0.004 & -0.070 & 0.005 & -0.060 & 0.004 \\
\hline & & & 1 & 1.5 & 0.125 & 0.031 & -0.063 & 0.004 & -0.071 & 0.006 & -0.061 & 0.004 \\
\hline & & & 1 & 2 & 0.167 & 0.056 & 0.033 & 0.001 & 0.035 & 0.002 & 0.026 & 0.001 \\
\hline & \multirow[t]{6}{*}{50} & \multirow[t]{3}{*}{10} & 0.5 & 1 & 0.019 & 0.001 & -0.076 & 0.006 & -0.082 & 0.007 & -0.075 & 0.006 \\
\hline & & & 1 & 1.5 & -0.083 & 0.014 & -0.027 & 0.001 & -0.031 & 0.001 & -0.026 & 0.001 \\
\hline & & & 1 & 2 & 0.041 & 0.003 & -0.043 & 0.002 & -0.039 & 0.002 & -0.038 & 0.002 \\
\hline & & \multirow[t]{3}{*}{25} & 0.5 & 1 & 0.081 & 0.013 & -0.014 & 0.001 & -0.008 & 0.000 & -0.006 & 0.000 \\
\hline & & & 1 & 1.5 & -0.109 & 0.024 & -0.033 & 0.001 & -0.027 & 0.001 & -0.025 & 0.001 \\
\hline & & & 1 & 2 & -0.010 & 0.000 & 0.111 & 0.013 & 0.109 & 0.012 & 0.103 & 0.011 \\
\hline \multirow[t]{12}{*}{0.75} & 30 & 5 & 0.5 & 1 & -0.036 & 0.003 & -0.125 & 0.016 & -0.128 & 0.017 & -0.135 & 0.019 \\
\hline & & & 1 & 1.5 & -0.015 & 0.000 & -0.054 & 0.003 & -0.059 & 0.004 & -0.068 & 0.005 \\
\hline & & & 1 & 2 & 0.008 & 0.000 & -0.100 & 0.011 & -0.103 & 0.011 & -0.111 & 0.013 \\
\hline & & 10 & 0.5 & 1 & -0.060 & 0.007 & -0.141 & 0.020 & -0.144 & 0.021 & -0.152 & 0.024 \\
\hline & & & 1 & 1.5 & 0.019 & 0.001 & -0.086 & 0.008 & -0.090 & 0.008 & -0.098 & 0.010 \\
\hline & & & 1 & 2 & -0.194 & 0.076 & 0.045 & 0.002 & 0.061 & 0.004 & 0.068 & 0.005 \\
\hline & 50 & 10 & 0.5 & 1 & 0.050 & 0.005 & -0.083 & 0.007 & -0.086 & 0.008 & -0.093 & 0.009 \\
\hline & & & 1 & 1.5 & -0.009 & 0.000 & -0.074 & 0.006 & -0.077 & 0.006 & -0.083 & 0.007 \\
\hline & & & 1 & 2 & -0.009 & 0.000 & 0.083 & 0.007 & 0.101 & 0.010 & 0.109 & 0.012 \\
\hline & & 25 & 0.5 & 1 & -0.036 & 0.003 & -0.069 & 0.005 & -0.073 & 0.006 & -0.081 & 0.007 \\
\hline & & & 1 & 1.5 & -0.015 & 0.000 & -0.092 & 0.009 & -0.095 & 0.010 & -0.103 & 0.011 \\
\hline & & & 1 & 2 & 0.083 & 0.014 & -0.097 & 0.010 & -0.101 & 0.011 & -0.108 & 0.012 \\
\hline
\end{tabular}

better than the corresponding MLE's in terms of bias and MSE but MLE's of $\alpha$ perform better than the corresponding Bayes estimators in terms of bias and MSE. Among the Bayes estimates of $\beta$, estimator under squared error loss function possess minimum bias and MSE. Also among the Bayes estimates of $p$, estimator under entropy loss functions possess minimum bias and MSE for small 
Table 5: Progressive type-II censoring samples with binomial removals

\begin{tabular}{|c|c|c|c|c|}
\hline Scheme & $p$ & $m$ & $R_{i}$ & Data \\
\hline 1 & .25 & 10 & $5,6,3,7,1,2,3,0,1,1$ & $0.5,0.7,1.1,1.5,3.0,4.0,4.7,7.0,7.5,9.0$ \\
\hline 2 & .25 & 15 & $9,5,1,2,3,0,2,1,1,1,0,0,0,0,0$ & $\begin{array}{l}0.5,1.0,1.5,1.5,2.2,3.0,3.3,4.5,5.0,5.4,7.5,8.8,9.0, \\
10.2,22.0\end{array}$ \\
\hline 3 & .25 & 20 & $\begin{array}{l}1,6,4,3,2,1,1,0,1,0,0,0,0,0,0,0,1 \\
0,0,0\end{array}$ & $\begin{array}{l}0.5,0.6,1.0,1.3,1.5,2.2,2.7,3.0,3.3,4.0,4.5,4.7,5.0 \text {, } \\
5.4,5.4,7.0,7.5,9.0,10.2,22.0\end{array}$ \\
\hline 4 & .5 & 10 & $14,9,4,1,2,0,0,0,0,0$ & $0.5,1.5,3.0,4.7,5.4,7.5,8.8,9.0,10.2,22.0$ \\
\hline 5 & 0.5 & 15 & $16,3,3,2,0,1,0,0,0,0,0,0,0,0,0$ & $\begin{array}{l}0.5,1.5,2.2,3.0,4.0,4.5,5.0,5.4,5.4,7.0,7.5,8.8,9.0 \\
10.2,22.0 \text {, }\end{array}$ \\
\hline 6 & 0.5 & 20 & $\begin{array}{l}9,4,2,3,1,1,0,0,0,0,0,0,0,0,0,0,0, \\
0,0,0\end{array}$ & $\begin{array}{l}0.5,1.0,1.5,1.5,2.5,3.0,3.3,4.0,4.0,4.5,4.7,5.0,5.4 \text {, } \\
5.4,7.0,7.5,8.8,9.0,10.2,22.0\end{array}$ \\
\hline 7 & 0.75 & 10 & $20,8,2,0,0,0,0,0,0,0$ & $0.5,2.2,4.7,5.4,7.0,7.5,8.8,9.0,10.2,22.0$ \\
\hline 8 & 0.75 & 15 & $17,6,2,0,0,0,0,0,0,0,0,0,0,0,0$ & $\begin{array}{l}0.5,1.5,3.0,4.0,4.5,4.7,5.0,5.4,5.4,7.0,7.5,8.8,9.0, \\
10.2,22.0\end{array}$ \\
\hline 9 & 0.75 & 20 & $\begin{array}{l}10,7,3,0,0,0,0,0,0,0,0,0,0,0,0,0,0, \\
0,0,0\end{array}$ & $\begin{array}{l}0.5,1.0,2.0,2.7,3.0,3.0,3.3,4.0,4.0,4.5,4.7,5.05 .4 \\
5.47 .0,7.5,8.8,9.0,10.2,22.0\end{array}$ \\
\hline
\end{tabular}

Table 6: Maximum likelihood estimates of $\alpha, \beta$ and $p$, Bayes estimates of $\alpha, \beta$ and $p$ based on data under squared error, LINEX and entropy loss functions for different choices of $p=0.25,0.5$ and 0.75 .

\begin{tabular}{|c|c|c|c|c|c|c|c|c|c|c|c|c|c|}
\hline$p$ & $m$ & $\hat{\alpha}$ & $\hat{\beta}$ & $\hat{p}$ & $\hat{\alpha}_{S B}$ & $\hat{\alpha}_{L B}$ & $\hat{\alpha}_{E B}$ & $\hat{\beta}_{S B}$ & $\hat{\beta}_{L B}$ & $\hat{\beta}_{E B}$ & $\hat{p}_{S B}$ & $\hat{p}_{L B}$ & $\hat{p}_{E B}$ \\
\hline \multirow[t]{3}{*}{0.25} & 10 & 1.120 & 0.253 & 0.243 & 0.014 & 0.014 & 0.003 & 0.048 & 0.047 & 0.043 & 0.255 & 0.254 & 0.248 \\
\hline & 15 & 1.228 & 0.279 & 0.214 & 0.018 & 0.018 & 0.005 & 0.062 & 0.062 & 0.058 & 0.227 & 0.226 & 0.221 \\
\hline & 20 & 1.033 & 0.202 & 0.253 & 0.016 & 0.015 & 0.004 & 0.073 & 0.073 & 0.068 & 0.267 & 0.266 & 0.258 \\
\hline \multirow[t]{3}{*}{0.5} & 10 & 1.101 & 0.212 & 0.508 & 0.011 & 0.011 & 0.002 & 0.041 & 0.041 & 0.037 & 0.500 & 0.498 & 0.493 \\
\hline & 15 & 1.409 & 0.263 & 0.446 & 0.015 & 0.015 & 0.002 & 0.059 & 0.059 & 0.054 & 0.444 & 0.442 & 0.435 \\
\hline & 20 & 1.493 & 0.213 & 0.444 & 0.018 & 0.017 & 0.004 & 0.076 & 0.076 & 0.071 & 0.443 & 0.441 & 0.432 \\
\hline \multirow[t]{3}{*}{0.75} & 10 & 1.383 & 0.227 & 0.750 & 0.018 & 0.018 & 0.005 & 0.047 & 0.047 & 0.042 & 0.701 & 0.699 & 0.694 \\
\hline & 15 & 1.937 & 0.275 & 0.714 & 0.019 & 0.018 & 0.002 & 0.061 & 0.061 & 0.055 & 0.668 & 0.665 & 0.660 \\
\hline & 20 & 2.110 & 0.221 & 0.606 & 0.019 & 0.018 & 0.002 & 0.075 & 0.075 & 0.070 & 0.575 & 0.572 & 0.564 \\
\hline
\end{tabular}

values of $p$ and squared error loss function possess minimum bias and MSE for large value of $p$. A real data was also used to illustrate the estimation procedures proposed in this paper.

\section{References}

Al-Zahrani, B. (2012), "Maximum likelihood estimation for generalized Pareto distribution under progressive censoring with binomial removals," Open Journal of Statistics, 2, 420.

Amin, Z. H. (2008), "Bayesian inference for the Pareto lifetime model under progressive censoring with binomial removals," Journal of Applied Statistics, 35, 1203-1217. 
Azimi, R., Fasihi, B., and Sarikhanbaglu, F. A. (2014), "Statistical inference for generalized Pareto distribution based on progressive Type-II censored data with random removals," International Journal of Scientific World, 2, 1-9.

Azimi, R. and Yaghmaei, F. (2013), "Bayesian estimation based on Rayleigh progressive type II censored data with binomial removals," Journal of Quality and Reliability Engineering, 2013.

Balakrishnan, N. and Aggarwala, R. (2000), Progressive censoring: theory, methods, and applications, Boston, Birkhauser and publisher.

Chacko, M. and Mohan, R. (2018), "Statistical Inference for Gompertz Distribution based on Progressive Type-II Censored Data with Binomial Removals," Statistica, 78, 251-272.

de Araujo Rodrigues, J. and Silva, A. P. C. M. (2015), "The exponentiated Kumaraswamyexponential distribution," British Journal of Applied Science \& Technology, 10, 1-12.

Elbatal, I. (2013), "Kumaraswamy linear exponential distribution," Pioneer J Theor Appl Statist, 5, $59-73$.

Feroze, N. and El-Batal, I. (2013), "Parameter Estimations Based On Kumaraswamy Progressive Type II Censored Data with Random Removals," Journal of Modern Applied Statistical Methods, $12,19$.

Jorgensen, B. (1982), Statistical properties of the generalized inverse Gaussian distribution, vol. 9, Springer Science \& Business Media.

Lawless, J. F. (2003), Statistical models and methods for lifetime data, John Wiley \& Sons, New York, 2nd ed.

Lemonte, A. J., Barreto-Souza, W., Cordeiro, G. M., et al. (2013), "The exponentiated Kumaraswamy distribution and its log-transform," Brazilian Journal of Probability and Statistics, $27,31-53$.

Miller, R. G. (1981), “Survival Analysis. John Willey \& Sons,” New York.

Mohammed, B. (2014), "Statistical properties of Kumaraswamy-Generalized exponentiated exponential distribution," International Journal of Computer Applications, 94.

Nadarajah, S., Cordeiro, G. M., and Ortega, E. M. (2012), "General results for theKumaraswamy-G distribution," Journal of Statistical Computation and Simulation, 82, 951-979.

Stephens, M. A. (1974), "EDF statistics for goodness of fit and some comparisons," Journal of the American statistical Association, 69, 730-737.

Tse, S. K., Yang, C., and Yuen, H.-K. (2000), "Statistical analysis of Weibull distributed lifetime data under Type II progressive censoring with binomial removals," Journal of Applied Statistics, 27, 1033-1043. 
Wu, C.-C., Wu, S.-F., Chan, H.-Y., et al. (2004), "MLE and the estimated expected test time for Pareto distribution under progressive censoring data," International journal of information and management sciences 15 (3), 29-42.

Wu, S.-J. and Chang, C.-T. (2003), "Inference in the Pareto distribution based on progressive Type II censoring with random removals," Journal of Applied Statistics, 30, 163-172.

Yuen, H.-K. and Tse, S.-K. (1996), "Parameters estimation for Weibull distributed lifetimes under progressive censoring with random removals," Journal of Statistical Computation and Simulation, $55,57-71$.

Received: February 15, 2019

Accepted: September 18, 2019 